\title{
Management of iron deficiency anaemia in secondary care across England between 2012 and 2018: a real-world analysis of Hospital Episode Statistics
}

\author{
Matthew James Brookes (D) , ${ }^{1,2}$ Angela Farr (D) , ${ }^{3}$ Ceri J Phillips, ${ }^{3}$ \\ Nigel John Trudgill ${ }^{4}$
}

- Additional material is published online only. To view, please visit the journal online (http://dx.doi.org/10.1136/ flgastro-2020-101506).

For numbered affiliations see end of article.

\section{Correspondence to}

Professor Matthew James Brookes, Gastroenterology, Royal Wolverhampton Hospitals NHS Trust, Wolverhampton WV10 OQP, UK; m.j.brookes@bham. ac.uk

Received 24 April 2020 Revised 14 September 2020 Accepted 6 October 2020 Published Online First 26 October 2020

\section{SLinked}

http://dx.doi.org/10.1136/ flgastro-2020-101695

\section{Check for updates}

(C) Author(s) (or their employer(s)) 2021. No commercial re-use. See rights and permissions. Published by BMJ.

To cite: Brookes MJ,

Farr A, Phillips CJ, et al.

Frontline Gastroenterology

2021;12:363-369.

\section{ABSTRACT}

Objective Iron deficiency anaemia (IDA) occurs in $2 \%-5 \%$ of men and postmenopausal women in the developed world and, if left untreated, can significantly impair quality of life or decompensate chronic illnesses. Approximately $10 \%$ of men and postmenopausal women with IDA have underlying gastrointestinal malignancy. This study identifies trends in the management of IDA in secondary care in England.

Design/method The Hospital Episode Statistics database was used to analyse IDA-related hospital and outpatient admissions (elective and non-elective) in National Health Service England between April 2012 and March 2018. Outcome measures included rates of readmission, length of stay (LOS) and cost per admission.

Results Between 2012/2013 and 2017/2018, there was a $72 \%$ increase in hospital admissions for patients with a primary diagnosis of IDA and a $68 \%$ increase in hospital spells, with the number of cases being managed non-electively increasing by $58 \%$. Non-electively managed patients had a longer LOS (3.10 vs 0.04 days, respectively) and increased rate of readmissions within 30 days $(24.1 \%$ vs $6.6 \%)$ versus patients managed electively. Average day-case cost was $£ 449$ versus f1676 for non-elective admission. Across the 195 clinical commissioning groups (CCGs) in England, non-elective spells per 100000 population demonstrated extensive and widening variability, ranging from 18 to 118 in 2017/2018 compared with 11-55 in 2012/2013.

Conclusion The current analysis highlights several opportunities to improve patient outcomes and reduce costs. There is an opportunity to improve day-case services by looking at the difference between CCGs and the variability in care and to reduce the number of non-elective admissions.

\section{Significance of this study}

What is already known on this topic

- Iron deficiency anaemia (IDA) occurs in $2 \%-5 \%$ of adult men and postmenopausal women in the developed world and, if left untreated, significantly impair quality of life or decompensate chronic illnesses.

- Approximately $10 \%$ of men and postmenopausal women with IDA have underlying gastrointestinal (GI) malignancy.

- Non-elective admissions for IDA has previously been described in over $9 \%$ of patients admitted to UK hospitals for IDA.

\section{What this study adds}

- The number of IDA cases being managed as non-elective emergency admissions increased by 58\% between 2012/2013 and 2017/2018.

- Patients managed via non-elective emergency admissions have longer length of stay (3.10 vs 0.04 days, respectively) and increased rate of readmissions within 30 days ( $24.1 \%$ vs $6.6 \%)$ vs patients managed electively.

- Considerable variability in IDA management exists within clinical commissioning groups across National Health Service England. Across the 195 CCGs in England, non-elective spells per 100000 population demonstrated extensive and widening variability, ranging from 18 to 118 in 2017/2018, compared with 11-55 in 2012/2013.

\section{INTRODUCTION}

Iron deficiency anaemia (IDA) occurs in $2 \%-5 \%$ of adult men and postmenopausal women in the developed world and, if left 


\section{Significance of this study}

\section{How might it impact on clinical practice in the} foreseeable future

- The current analysis highlights a number of service improvements that could be made to improve patient outcomes and reduce costs, reducing non-elective emergency admissions with a primary diagnosis of IDA, thereby reducing 30-day readmission following nonelective emergency admissions and transferring IDA treatment from the more costly non-elective admission to day-case treatment.

untreated, significantly impair quality of life or decompensate chronic illnesses. ${ }^{12}$ Iron deficiency can occur, with or without anaemia, when iron availability is insufficient to meet the body's needs. ${ }^{13}$ Although IDA may be diagnosed with appropriate additional blood tests and treated with either dietary replacement, oral or intravenous iron, this condition is frequently overlooked. ${ }^{2-5}$

Reducing unplanned hospitalisation in individuals with ambulatory care sensitive conditions (ACSC), such as IDA, is a key indicator in the National Health Service (NHS) Outcomes Framework for improving the quality of life of patients with long-term conditions $^{5}$; nonetheless, IDA is the seventh most preventable cause for hospital admissions (ie, higher than asthma or diabetes). ${ }^{4}$

Individuals at high risk of IDA include those with chronic inflammation (eg, inflammatory bowel disease, chronic heart failure and chronic kidney disease) and presurgery or blood loss (eg, heavy menstrual bleeding). ${ }^{13}$ IDA may also occur with numerous upper and lower gastrointestinal (GI) pathologies ${ }^{1}$; thus, IDA is a common cause of referral to gastroenterologists, representing 4\%-13\% of all gastroenterology referrals. ${ }^{2}{ }^{6}$ From these cases, approximately $10 \%$ of men and postmenopausal women with IDA have underlying GI malignancy, often in the absence of any other clinical manifestations. ${ }^{7}$ Thus, IDA is recognised as an urgent indication for GI investigation and delayed/incomplete investigations for underlying GI pathology carry the potential for GI cancers to be missed. $^{7}$

The current British Society for Gastroenterology (BSG) guidelines suggest that 'all patients (other than menstruating women) with IDA and no obvious cause should have both an upper GI endoscopy and either colonoscopy or radiological imaging (unless carcinoma or coeliac disease is found)' ${ }^{2}$

The objective of the current study was to use realworld data from the English NHS to identify trends between 2012 and 2018 in the management of IDA in secondary care, including financial and in-hospital costs.

\section{METHODS}

Data source and study data

Hospital admissions relating to IDA between April 2012 and March 2018 were analysed using the Hospital Episode Statistics (HES) database, which contains details of all admissions to NHS hospitals and all NHS outpatient appointments in England ${ }^{8}$; HES data are provided by the NHS Information Centre for Health and Social Care under a commercial reuse licence via Harvey Walsh Ltd. Data were pseudoanonymised to prevent individual patient identification.

HES data for patients with a diagnosis of IDA were based on postdischarge diagnostic coding from secondary care and align to the following International Classification of Disease codes (10th iteration): D50.0 (IDA secondary to blood loss (chronic)), D50.8 (other IDA) and D50.9 (IDA, unspecified).

Based on the previous codes, data were requested for patients with or without IDA and segmented into yearly intervals. National data were segmented into clinical commissioning groups (CCGs) for the year 2017/2018. Admission data for elective and nonelective patients and spells were included. To avoid skewing diagnosis with coding practice, the data were split into three main groups: patients with IDA coded in the primary position, patients with IDA coded in the secondary position and patients with IDA coded in any position. Unless otherwise specified, the results refer to patients with IDA coded in the primary position.

\section{Outcome measures}

Outcome measures including rates of readmission within 30 days, bed days, length of stay (LOS) and cost per admission were collected. Prescription data for oral ferrous iron products were also included. CCG prescription data were analysed for the community setting (not the hospital setting).

\section{Statistical evaluation}

Descriptive statistics were used to analyse the data and, where appropriate, quantitative variables were used according to mean and range. Qualitative variables were described according to the absolute and/or relative (\%) frequency of each class. Missing data were not considered in the calculation of percentages. Correlations were analysed using Pearson product moment correlation coefficient (r), with results between -0.1 and 0.1 being deemed to have no correlation. Statistical significance was demonstrated for results $<0.05$ using Student's t-distribution, two-tailed test. To test statistical significance between correlation coefficients, Steiger's z-test was used. Statistical significance was also deemed from values $<0.05$.

\section{RESULTS}

\section{Primary diagnosis}

During the period April 2017 to March 2018, a total of 97781 patients and 159400 patients, respectively, 

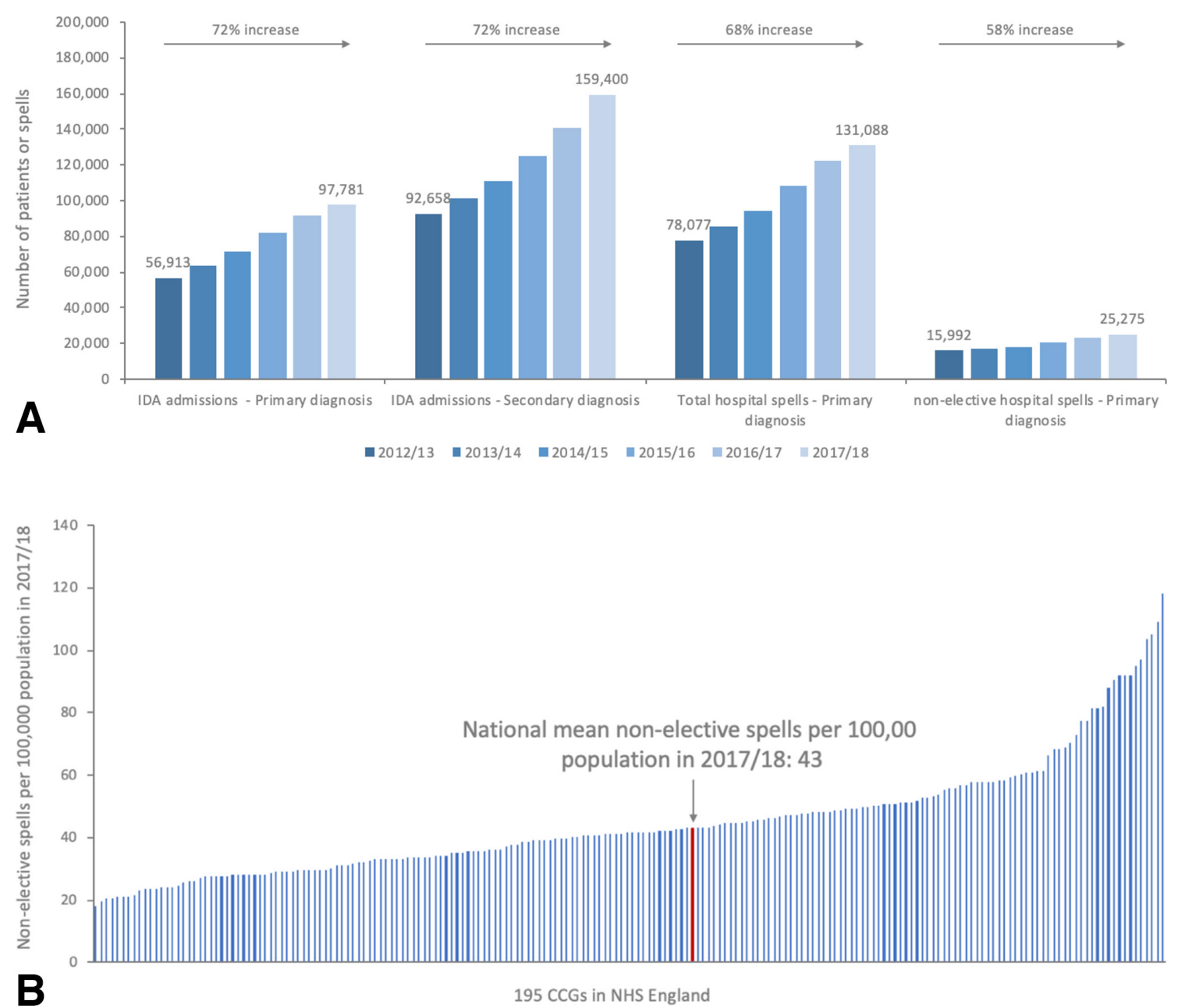

Figure 1 Analysis of (A) hospital admissions and hospital spells (total and non-elective) for patients with a primary/secondary diagnosis of IDA between 2012/2013 and 2017/2018 and (B) non-elective hospital spells (primary diagnosis of IDA) per 100000 population across all CCGs in England 2017/2018. Each vertical line represents an individual CCG, with the bold red line representing the national mean for England. CCG, clinical commissioning groups; IDA, iron deficiency anaemia.

had a primary and a secondary diagnosis of IDA, with the former accounting for 131088 hospital admissions in England (figure 1A). These data represent a $72 \%$ increase between 2012/2013 and 2017/2018 in patients admitted to hospital with either a primary or secondary diagnosis of IDA, and a $68 \%$ increase in hospital admissions (spells) since 2012/2013 for those admitted with a primary diagnosis (figure 1A). Nonetheless, year-on-year data for hospital spells per patient remained relatively constant (average of 1.37 in $2012 / 2013$; 1.34 in $2013 / 2014$; 1.32 in $2014 / 2015$; 1.33 in 2015/2016; 1.34 in 2016/2017; and 1.34 spells per patient in 2017/2018.

\section{Non-elective spells for patients with a primary diagnosis of IDA}

Between 2012/2013 and 2017/2018, there was a $58 \%$ increase in the number of non-elective spells, accounting for 9283 additional spells (figure 1A). Assuming a population of 58719921 in England, this equates to 43.0 non-elective spells per 100000 population during the period 2017/2018, compared with
29.3 non-elective spells per 100000 population in 2012/2013.

Correlation analysis to explore the relationship between demographic factors and non-elective spells only showed a correlation between higher rates of non-elective admissions and increasing levels of deprivation $(\mathrm{r}=-0.233, \mathrm{p}=0.001)$, while no correlation was seen for age (\% patients $\geq 65: r=0.015, p=0.837$ ) or gender (\% female patients: $r=0.105, p=0.144)$.

Comparison of non-elective spells per 100000 population across CCGs in England demonstrated wide variation (eg, range in 2017/2018: 18-118 nonelective spells per 100000 population; figure 1B). We also noted a widening variation between CCGs in 2017/2018 compared with 2012/2013 (range: 11-55 non-elective spells per 100000 population).

\section{Cost burden of IDA admissions}

The increasing number of patients admitted to hospital with a primary diagnosis of IDA contributed to a $38 \%$ increase in total expenditure for managing IDA in 

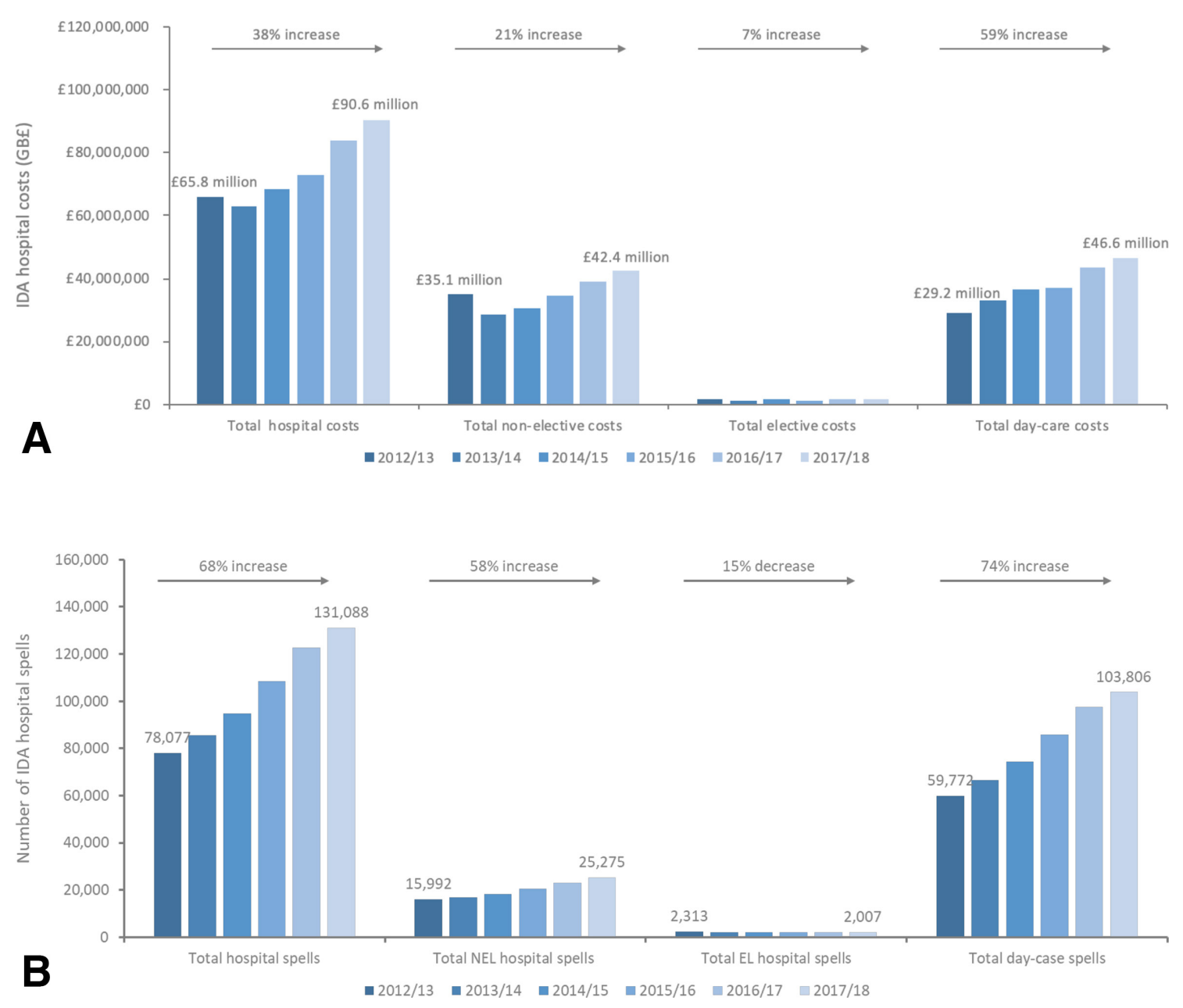

Figure 2 Annual (A) costs of admissions and (B) number of hospital spells, for patients with a primary diagnosis of IDA between 2012/2013 and 2017/2018. EL, elective; IDA, iron deficiency anaemia; NEL, non-elective .

England ( $£ 65.8$ million in $2012 / 2013$ to $£ 90.6$ million in 2017/2018; figure 2A). Day-case costs increased by $59 \%$ from $£ 29.2$ million in $2012 / 2013$ to $£ 46.6$ million in 2017/2018, while non-elective expenditure increased by $21 \%$ from $£ 35.1$ million in $2012 / 2013$ to $£ 42.4$ million in $2017 / 2018$.

Thus, day-case treatment accounted for the majority ( $£ 17.3$ million, equating to $70 \%$ ) of the increase in total costs between 2012/2013 and 2017/2018, with non-elective accounting for $29 \%$ ( $£ 7.2$ million) and EL admissions accounting for $1 \%$.

Total annual costs in 2017/2018 for day case (103 806) were managed at a cost of $£ 46.5$ million. Total annual costs in 2017/2018 for non-elective case (25 $275)$ was $£ 42.3$ million; while these are not directly comparable costs, it does represent a fourfold difference in the number of patients treated (figure 2).

Considerable variation from the national average for total cost per 100000 population of $£ 154248$ was observed within CCGs across England in 2017/2018 (total cost range per 100000 population: $£ 71$ $259-£ 345114$; online supplemental figure 1 ).

\section{Length of stay}

Although elective admissions in 2017/2018 accounted for $94 \%$ of bed days (78 292 elective bed days) and only $6 \%$ days were due to non-elective admissions (4623 non-elective bed days), the average LOS was considerably longer for an non-elective spell than an elective spell (3.10 vs 0.04 days, respectively; table 1).

Minor LOS reductions were observed between $2012 / 2013$ and $2017 / 2018$, with a $21.6 \%$ reduction when IDA was the primary diagnosis (1.8 to 1.4 days; table 1) and an $11.4 \%$ reduction when IDA was a secondary diagnosis (7.4 days to 6.6 days). Furthermore, in patients with a primary IDA diagnoses, LOS due to non-elective have been steadily reducing, while LOS due to elective admissions have remained relatively constant (online supplemental figure 2). There was wide variation in LOS within CCGs in England in 2017/2018 (range: 0.44 days-9.46 days).

\section{Readmissions with IDA}

Non-elective patients had approximately a threefold higher chance of readmission within 30 days than elective and day-case patients (eg, $24.1 \%$ vs 
Table 1 Bed days, LOS and readmissions (within 30 days of original spell)* for patients with a primary diagnosis of IDA between 2012/2013 and 2017/2018

\begin{tabular}{|c|c|c|c|c|c|c|c|c|c|}
\hline HES year/date range & $\begin{array}{l}\text { Total bed } \\
\text { days }\end{array}$ & $\begin{array}{l}\text { Total non-elective } \\
\text { bed days }\end{array}$ & $\begin{array}{l}\text { Total elective } \\
\text { bed days }\end{array}$ & $\begin{array}{l}\text { Mean LOS } \\
\text { total }\end{array}$ & $\begin{array}{l}\text { Mean LOS non- } \\
\text { elective spells }\end{array}$ & $\begin{array}{l}\text { Mean LOS } \\
\text { elective spells }\end{array}$ & $\begin{array}{l}\% \text { non-elective } \\
\text { readmitting }\end{array}$ & $\begin{array}{l}\% \text { elective/DC } \\
\text { readmitting }\end{array}$ & $\begin{array}{l}\text { Difference between } \\
\% \text { non-elective and } \% \\
\text { elective/day case }\end{array}$ \\
\hline $2012 / 2013$ & 73929 & 5495 & 68434 & 1.80 & 4.28 & 0.09 & 22.4 & 6.4 & 15.9 \\
\hline $2013 / 2014$ & 72464 & 4370 & 68094 & 1.65 & 4.05 & 0.06 & 21.5 & 6.1 & 15.4 \\
\hline $2014 / 2015$ & 75076 & 5070 & 70006 & 1.58 & 3.86 & 0.07 & 22.5 & 6.2 & 16.3 \\
\hline $2015 / 2016$ & 80498 & 4629 & 75869 & 1.50 & 3.70 & 0.05 & 23.2 & 6.4 & 16.7 \\
\hline $2016 / 2017$ & 85254 & 5502 & 79752 & 1.41 & 3.47 & 0.06 & 24.0 & 6.5 & 17.5 \\
\hline $2017 / 2018$ & 82915 & 4623 & 78292 & 1.41 & 3.10 & 0.04 & 24.1 & 6.6 & 17.5 \\
\hline $\begin{array}{l}\text { Difference between } \\
2012 / 2013 \text { and } \\
2017 / 2018\end{array}$ & 8986 & -872 & 9858 & -0.39 & -1.18 & -0.04 & - & - & - \\
\hline $\begin{array}{l}\% \text { change between } \\
2012 / 2013 \text { and } \\
2017 / 2018\end{array}$ & 12.2 & -15.9 & 14.4 & -21.6 & -27.6 & -50.6 & 1.7 & 0.2 & 1.6 \\
\hline
\end{tabular}

$6.6 \%$, respectively, in 2017/2018; table 1). Between $2012 / 2013$ and 2017/2018, there was a slight increase in readmission rates for both non-elective patients $(22.4 \%$ and $24.1 \%$, respectively) and elective and day-case patients $(6.4 \%$ and $6.6 \%$; table 1$)$. Additionally, in $2017 / 2018$, there was substantial variation in the numbers of patients readmitted within 30 days following a non-elective admission between CCGs (range: 15.2\%-37.0\%).

\section{Management of IDA}

Between 2012/2013 and 2017/2018, prescriptions for community-prescribed oral iron increased by 1313 per 100000 population (10.7\% more) at an increased cost of $£ 14537$ (66.0\% more; online supplemental table 1). Higher rates of oral iron prescriptions in the community correlated with a higher frequency of non-elective admissions for IDA in CCGs in England (online supplemental figure 3).

Meanwhile, analysis assessing the correlation between oral iron prescriptions for IDA and nonelective and day-case admissions per 100000 population indicated that prior treatment with oral iron did not prevent non-elective admissions $(r=0.284$, $\mathrm{p}<0.001)$ or day-case admissions $(\mathrm{r}=0.201$, $\mathrm{p}<0.005)$.

The most widely prescribed oral iron was ferrous fumarate, with prescriptions and accompanying costs increasing by $28 \%$ and $124 \%$, respectively, between 2012/2013 and 2017/2018 (online supplemental figure 4a,b). Meanwhile, overall prescriptions for intravenous iron decreased by 2.57 per 100000 population (56.4\% reduction), at a reduced cost of $£ 84.06$ per 100000 population $(54.8 \%$ reduction; online supplemental figure $4 \mathrm{c}, \mathrm{d})$.

The number of blood transfusions was also seen to increase year-on-year between 2012/2013 ( $n=1981)$ and 2017/2018 ( $\mathrm{n}=3271$; online supplemental table $1)$.

\section{DISCUSSION}

Using real-world data from the English NHS, the current analysis sought to explore trends in the secondary care management of IDA between 2012 and 2018. HES data for that period demonstrated a $72 \%$ increase in hospital admissions and 68\% increase in hospital spells for patients with a primary diagnosis of IDA, both of which contributed to increased expenditure for management of IDA in England. Although increasing year-on-year costs associated with IDA management were driven by both day-case and nonelective admissions, it is notable that with a similar budget, four times as many patients were managed as day cases compared with non-elective admissions. Despite the evidence that day-case treatment represents a more cost-effective means of managing IDA than non-elective admissions, there was a 58\% increase in non-elective admissions for patients with a primary diagnosis of IDA between 2012 and 2018.

Compared with patients managed electively, nonelective patients had considerably longer LOS (3.10 vs 0.04 days, respectively) and increased rates of 30 -day readmissions $(24.1 \%$ vs $6.6 \%)$ - both of which are key cost drivers for IDA management. Indeed, a number of studies have highlighted knowledge gaps regarding optimal management of patients presenting to emergency departments with IDA. ${ }^{9} 10$ Thus, transferring IDA management from non-elective to elective represents an important opportunity to reduce the financial burden of IDA, particularly with regards to extended LOS and increased readmissions, in an era when there is increasing financial pressure on healthcare systems.

Examination of non-elective admission rates across CCGs showed increasing non-elective admissions, as well as widening variation between CCGs, highlighting the need for standardised care pathways for IDA in England. The 2013 QualityWatch 'Focus on preventable admissions' report demonstrated that 
demographic factors, such as the level of deprivation, age and gender balance, can have an influence on inter-CCG variation in admission rates for ACSCs. ${ }^{11}$ However, the results of the current analysis only showed a correlation between non-elective admissions and deprivation levels, while gender and age were not correlated. A cause for this may lie in the introduction of streamlined organisational pathways or guidelines that have helped reduce non-elective admissions. Reducing non-elective admissions across all CCGs to the national average (or below) has the potential to improve patient outcomes, reduce costs and free up bed space, which is at a premium in English hospitals. ${ }^{12}$

In terms of trends in the management of IDA, prescriptions for oral and intravenous iron preparations and the number of blood transfusions increased during the period of our analyses, and this may represent an increased awareness of this condition and the need for treatment. Newer preparations of oral iron, including ferric maltol, are now available but were not included in the analysis in this study as they were only approved for limited use during the study period. Future studies will need to consider the usage of ferric maltol in the treatment of IDA in selected situations. ${ }^{13}$ Current BSG guidelines recommend that all patients have iron supplementation to correct anaemia and replenish body stores, while parenteral iron may be used when oral preparations are not tolerated and blood transfusions should be reserved for patients with or at risk of cardiovascular instability due to the degree of their anaemia. ${ }^{2}$ Whether the use of parenteral iron would have an impact on non-elective admission rates is uncertain and was not analysed in this manuscript due to the relatively small number of infusions seen. Future studies might consider evaluating the impact of parenteral iron in these settings to establish if there were a role in the prevention of non-elective admissions.

To our knowledge, this is the largest observational analysis of IDA management within the English NHS. Although HES represents the most comprehensive source of healthcare data available within the UK, there are limitations to these data, namely that the quality of the data is dependent on the accuracy and completeness of the clinical coding. ${ }^{14}$ However, HES data are analysed and audited regularly to ensure consistency and compliance to national regulations, and the current analysis was performed based on the largest and most appropriate available dataset relating to secondary care management of IDA in England.

The results of the current analysis highlight a number of potential opportunities to improve patient outcomes, as well as reduce IDA management costs. These include reducing non-elective emergency admissions for individuals with a primary diagnosis of IDA, thereby reducing readmission within 30 days following non-elective emergency admission and transferring IDA treatment from the more costly non-elective admission to day-case treatment. In addition, the wide variability in standards of care across CCGs highlights the need for collaborative ambulatory care pathways for IDA management in England.

To reduce variance across CCGs, learning from centres where the non-elective admission rates are low and sharing best practice could support the adoption of successful IDA management and care pathways. More can be done to reduce non-elective admissions and real-word data such as this can inform and improve management, care and potentially NHS budgets.

\section{Author affiliations}

${ }^{1}$ Department of Gastroenterology, Royal Wolverhampton Hospitals NHS Trust, Wolverhampton, UK

${ }^{2}$ Research Institue, University of Wolverhampton Faculty of Science and Engineering, Wolverhampton, UK

${ }^{3}$ College of Human and Health Sciences, Swansea University, Swansea, UK ${ }^{4}$ Department of Gastroenterology, Sandwell General Hospital, West Bromwich, UK

Correction notice This article has been corrected since it published Online First. The second author's name has been amended.

Contributors MJB and CJP developed the initial research question and project protocol and then contributed to data analysis and preparation of the manuscript. AF assisted with data analysis and preparation of the manuscript. NJT contributed to data analysis and interpretation and manuscript preparation. All authors contributed to the writing and preparation and approval of the final manuscript. All authors had access to all of the study data and can take responsibility for data integrity and the accuracy of analyses. AF and CJP conducted the statistical analysis. MJB undertook primary drafting of the paper, and all authors contributed to significant redrafting. MJB is the guarantor of the paper.

Funding Vifor Pharma UK Ltd funded unrestricted access to the Hospital Episode Statistics (HES) data through Harvey Walsh Ltd.

Competing interests Manuscript was produced with the support of data accessed through HES and funded by Vifor International. MJB: Vifor International funded expenses and honoraria associated with analysis and manuscript preparation. CJP: Vifor International funded expenses and honoraria associated with analysis and manuscript preparation. MJB, NT and CJP have previously received consultancy fees from Vifor.

Patient consent for publication Not required.

Provenance and peer review Not commissioned; externally peer reviewed.

Data availability statement Data are available on reasonable request. Data are accessed from HES data and are provided by the National Health Service Information Centre for Health and Social Care under a commercial reuse licence via Harvey Walsh Ltd.

Supplemental material This content has been supplied by the author(s). It has not been vetted by BMJ Publishing Group Limited (BMJ) and may not have been peer-reviewed. Any opinions or recommendations discussed are solely those of the author(s) and are not endorsed by BMJ. BMJ disclaims all liability and responsibility arising from any reliance placed on the content. Where the content includes any translated material, BMJ does not warrant the accuracy and reliability of the translations (including but not limited to local regulations, clinical guidelines, terminology, drug names and drug dosages), and is not responsible for any error and/or omissions arising from translation and adaptation or otherwise.

\section{ORCID iDs}

Matthew James Brookes http://orcid.org/0000-0002-87820292 
Angela Farr http://orcid.org/0000-0002-2087-9310

\section{REFERENCES}

1 Abbaspour N, Hurrell R, Kelishadi R. Review on iron and its importance for human health. J Res Med Sci 2014;19:164-74.

2 Goddard AF, James MW, McIntyre AS, et al. Guidelines for the management of iron deficiency anaemia. Gut 2011;60:130916.

3 Cappellini MD, Comin-Colet J, de Francisco A, et al. Iron deficiency across chronic inflammatory conditions: international expert opinion on definition, diagnosis, and management. Am J Hematol 2017;92:1068-78.

4 Data on file: Hospital episode statistic data 2012-2018 (accessed under a commercial re-use licence via Harvey Walsh LTD.).

5 Department of Health. The NHS outcomes framework 2015/2016. Available: https://assets.publishing.service.gov. uk/government/uploads/system/uploads/attachment_data/file/ 385749/NHS_Outcomes_Framework.pdf [Accessed Jan 2020].

6 McIntyre AS, Long RG. Prospective survey of investigations in outpatients referred with iron deficiency anaemia. Gut 1993;34:1102-7.

7 Snook J. Investigating for Gi malignancy in iron-deficiency anaemia-the case for risk stratification. Frontline Gastroenterol 2014;5:229-30.
8 NHS Digital. Hospital episode statistics (Hes). Available: https://digital.nhs.uk/data-and-information/data-tools-andservices/data-services/hospital-episode-statistics [Accessed Jan 2020].

9 Boone S, Powers JM, Goodgame B, et al. Identification and management of iron deficiency anemia in the emergency department. J Emerg Med 2019;57:637-45.

10 Spradbrow J, Lin Y, Shelton D, et al. Iron deficiency anemia in the emergency department: over-utilization of red blood cell transfusion and infrequent use of iron supplementation. CJEM 2017;19:167-74.

11 Nuffield Trust. The health Foundation. QualityWatch: Focus on preventable admissions, 2013. https://www.health.org.uk/ publications/qualitywatch-focus-on-preventable-admissions

12 NHS England and NHS Improvement. Refreshing NHS plans for 2018/2019. Available: https://www.england.nhs.uk/ wp-content/uploads/2018/02/planning-guidance-18-19.pdf [Accessed Jan 2020].

13 Gasche C, Ahmad T, Tulassay Z, et al. Ferric maltol is effective in correcting iron deficiency anemia in patients with inflammatory bowel disease: results from a phase- 3 clinical trial program. Inflamm Bowel Dis 2015;21:579-88.

14 Nouraei SAR, Virk JS, Hudovsky A, et al. Accuracy of clinician-clinical coder information handover following acute medical admissions: implication for using administrative datasets in clinical outcomes management. J Public Health 2016;38:352-62. 